\title{
Force feedback facilitates multisensory integration during robotic tool use
}

\author{
Ali Sengül · Giulio Rognini • Michiel van Elk • \\ Jane Elizabeth Aspell · Hannes Bleuler · Olaf Blanke
}

Received: 21 November 2012 / Accepted: 9 April 2013 / Published online: 27 April 2013

(C) Springer-Verlag Berlin Heidelberg 2013

\begin{abstract}
The present study investigated the effects of force feedback in relation to tool use on the multisensory integration of visuo-tactile information. Participants learned to control a robotic tool through a surgical robotic interface. Following tool-use training, participants performed a crossmodal congruency task, by responding to tactile vibrations applied to their hands, while ignoring visual distractors superimposed on the robotic tools.
\end{abstract}

Electronic supplementary material The online version of this article (doi:10.1007/s00221-013-3526-0) contains supplementary material, which is available to authorized users.

A. Sengül $(\bowtie) \cdot$ G. Rognini $\cdot$ M. van Elk $\cdot$ O. Blanke

Center for Neuroprosthetics, School of Life Sciences, Ecole

Polytechnique Fédérale de Lausanne (EPFL), MAIF 02,

Ex bât.Maillefer, Technoparc, Rte du Bois 37, 1015 Lausanne,

Switzerland

e-mail: ali.sengul@epfl.ch

A. Sengül · G. Rognini · M. van Elk · J. E. Aspell · O. Blanke Laboratory of Cognitive Neuroscience, Brain Mind Institute,

Ecole Polytechnique Fédérale de Lausanne (EPFL), Lausanne, Switzerland

\section{A. Sengül · G. Rognini · H. Bleuler}

Robotic Systems Laboratory, Ecole Polytechnique Fédérale de

Lausanne (EPFL), Lausanne, Switzerland

M. van Elk

Department of Psychology, University of Amsterdam,

Amsterdam, The Netherlands

J. E. Aspell

Department of Psychology, Anglia Ruskin University,

Cambridge, UK

O. Blanke

Department of Neurology, University Hospital of Geneva (HUG),

Geneva, Switzerland
In the first experiment it was found that tool-use training with force feedback facilitates multisensory integration of signals from the tool, as reflected in a stronger crossmodal congruency effect with the force feedback training compared to training without force feedback and to no training. The second experiment extends these findings by showing that training with realistic online force feedback resulted in a stronger crossmodal congruency effect compared to training in which force feedback was delayed. The present study highlights the importance of haptic information for multisensory integration and extends findings from classical tool-use studies to the domain of robotic tools. We argue that such crossmodal congruency effects are an objective measure of robotic tool integration and propose some potential applications in surgical robotics, robotic tools, and human-tool interaction.

Keywords Multisensory integration - Crossmodal congruency effect $\cdot$ Force feedback $\cdot$ Robotic surgery

\section{Introduction}

The brain's body representation is characterized by lifelong plasticity that can be altered by experience. For instance, several experiments have revealed that fake or virtual hands as well as tools can be integrated in the body representation by investigating changes in behavior, neural activity, or the effects of focal brain damage on tool use (e.g., Iriki et al. 1996; Graziano et al. 2000; Graziano 1999). Changes in body representation have been observed following the use of relatively simple tools, such as a rake (Iriki et al. 1996; Maravita et al. 2002; Farne et al. 2005), and have been associated with regions in the posterior parietal and the premotor cortex in which bimodal neurons can be found that 
process visual and tactile cues (Iriki et al. 1996; Graziano et al. 1999; Graziano and Gandhi 2000). In an influential neurophysiological study it has been shown that after tool use the size of the visual receptive field of bimodal neurons in monkey's premotor and parietal cortex changed, often expanding to include the tip of the entire length of the tool (Iriki et al. 1996).

The effects of tool use and in particular the question of whether peripersonal space (i.e., the space around us that is within reach) is extended by tool use have been studied extensively using the crossmodal congruency task. In this task, participants are required to respond to tactile stimuliusually delivered to the thumb and index finger-while ignoring visual distractors presented in spatial proximity. The CCE is defined as the performance difference (reaction time or accuracy) between incongruent and congruent visuo-tactile stimulations and is typically larger when the visual and tactile stimuli are closer to each other in space (Spence et al. 2004). Visuo-tactile CCEs have been proposed to reflect the perceived proximity between visual and tactile stimuli and thereby provide an objective measure of the ease whereby multimodal information is integrated in the body representation (Zopf et al. 2010; Pavani et al. 2000; Aspell et al. 2009). Using the CCE, it has been found that active tool use increases the interference of visual stimuli presented at the tip of tools with vibrotactile stimuli presented on the hand that holds the tool (Maravita et al. 2002). This result was interpreted as reflecting that tools extended peripersonal space and that actively crossing the tools resulted in a remapping of peripersonal space (i.e., visual distractors presented at the end of a crossed tool interact with the hand that is holding the tool). These studies suggest that active tool use changes the neural representations of multisensory peripersonal space (but see Holmes et al. 2004).

Most studies on tool use and changes in the body representation have been carried out with physical tools, such as children's golf clubs (Holmes et al. 2004; Maravita et al. 2002) and a cane (Serino et al. 2007), or with pointing tools such as a computer mouse (Bassolino et al. 2010). Interestingly, a recent study suggests that virtual tool use relies on a similar mechanism as the use of real-world tools (Sengül et al. 2012). In recent years the use of virtual and robotic tools has increased dramatically. For example, more and more surgeons interact daily with robotic devices that allow them to perform complex surgical procedures in a more accurate and efficient manner without being in direct contact with the patient (Horgan and Vanuno 2001; Ayav et al. 2004; Maeso et al. 2010). In such systems, surgeons are seated in front of a console and can remotely control different robotic devices and tools that perform the surgical intervention inside the patient's body. The use of such teleoperated robotic systems thus separates the direct contact between surgeons and the biological tissue that the surgeon manipulates, but also removes the sense of touch. As a consequence, surgeons need to rely exclusively on visual cues to "feel" the tissue, which raises questions about the usability, intuitiveness and cognitive load of surgical robotic systems. Force feedback (FFB) has been shown to increase the usability and to decrease the cognitive workload during the surgery, as reflected in faster learning curves (Narazaki et al. 2006; Okamura 2009). In previous studies the absolute identification paradigm (AIP) and Fitts's law have been used to characterize the human-interface interaction (Tan et al. 2010; Zimmerli et al. 2012). These studies focused on specific surgical tasks and their completion during surgical procedures. However, it remains unknown whether FFB would also facilitate the integration of the tool in the body representation, as in the case of real-world tools. The integration of the tool in the body representation may be critical for a successful interaction with the surrounding world, as evidenced for instance by studies with upper limb amputees wearing a prosthesis. Thus, it has been found that sensory feedback is an important predictor of the success rate of training with the prosthesis. For instance, tactile feedback improved the integration of the prosthesis in the body representation, and as a consequence, the prosthesis was actually considered as a real and useful part of the body (Rosen et al. 2009; Marasco et al. 2011; Ehrsson et al. 2008).

Tool incorporation is a complex process that involves the integration of vision, touch, proprioception and motor cues. Analyzing the effect of each of these factors individually and systematically would provide a better understanding of the complex process of tool use. Haptic interfaces provide several advantages in this respect: They are robotic systems, which enable human users to touch and interact with virtual objects (Stanney et al. 1998; Mahvash and Hayward 2004) and offer the possibility of performing controlled studies with very precisely defined and standardized conditions in virtual reality that cannot be done in the real world. These devices thereby enable the independent manipulation of the different factors involved in tool use, such as FFB. Using a haptic device enables us to isolate the effect of FFB and to study its role in the multisensory integration of peripersonal space as measured by the crossmodal congruency task. The aim of the present study was to investigate the effect of FFB on tool incorporation as measured by the CCE. More specifically, we investigated whether visuo-tactile CCEs could be used as an objective assessment of the effects of FFB on tool use; as such, findings could inform surgery robotics to enhance safety and to increase the usability of the surgical robotic systems.

To this end, we used a new haptic device and virtual reality platform (see also Sengül et al. 2012) to investigate visuo-tactile integration as measured by the $\mathrm{CCE}$, during surgical-like tasks. We first tested whether and how the CCE was modulated by movements of the tool and FFB 
(study 1 and 2). Second, we tested whether a temporal mismatch between visual feedback and FFB would modulate the strength of the CCE (study 2).

\section{Materials and methods}

\section{Subjects}

A total of 20 healthy right-handed participants took part in the present experiments: twelve participants (three female) with an average age of 27.5 years (range 21-37 years) in study 1 and eight participants (four female) with an average age of 21.75 years (range 19-24 years) in study 2. All participants had normal or corrected to normal vision, no disorder of touch and had no history of neurological or psychiatric conditions. Each experiment took around 60 min per participant. Participants were informed about the general purpose of the research, gave their informed consent and were compensated for participating in the experiment. The study protocol was approved by the local ethics research committee-La Commission d' éthique de la recherche Clinique de la Faculté de Biologie et de Médecine-at the University of Lausanne, Switzerland, and was performed in accordance with the ethical standards described in the Declaration of Helsinki.

\section{Materials and apparatus}

In this study the same robotic system was used as described in Sengül et al. (2012). In this system the tracking of the hand movement and FFB are provided through a cabledriven bimanual haptic interface, thereby providing a large workspace for moving the hands. The system provides $7^{\circ}$ of freedom in motion, and it can render high forces in 3-translation directions ( $X Y Z)$ without instability. The system has two lightweight grippers that enable transparent interactions with virtual reality, thereby enabling realistic bimanual manipulations.

The CCE was measured using the same apparatus and procedures as in Sengül et al. (2012). The participants sat at a table and held two grippers (see Fig. 1). Their movement was transmitted by the encoders on the haptic device directly to the robotic tools so that they could interact with a deformable object and could see this interaction via the head-mounted display (HMD). CHAI 3D open-source platform and a set of $\mathrm{C}++$ libraries were used for modeling and simulating the haptics and for visualization of the virtual world. This platform supports several commercial haptic devices, and it is possible to extend it to support new custom-made FFB devices. Accordingly, we extended our platform by adding the drivers and libraries of our own customized FFB device (chai3d.org, Conti et al. 2005). A virtual world with two surgical tools and a deformable object was developed. The da Vinci surgical tools were modeled by a CAD program, Unigraphics, and imported into the virtual scene. To model the deformable object and interaction, GEL dynamics engine (Stanford University) was used. This engine enabled us to simulate the dynamic behavior of complex deformable objects (Parikh et al. 2009). The model used a skeleton of a mesh object filled with spheres that were connected with elastic links to model elongation,
Fig. 1 Experimental setup used in the experiments. A cabledriven haptic device (the $\mathrm{Da}$ Vinci simulator) with a large workspace was used. Participants interacted with the virtual object through the handles of the device, and their movements were shown through a HMD. To mask the noise of the vibrators and environment noise, headphones were used to present white noise. Participants responded to vibrotactile stimuli using the foot pedals. A chin rest system was used to prevent undesired movement of the head
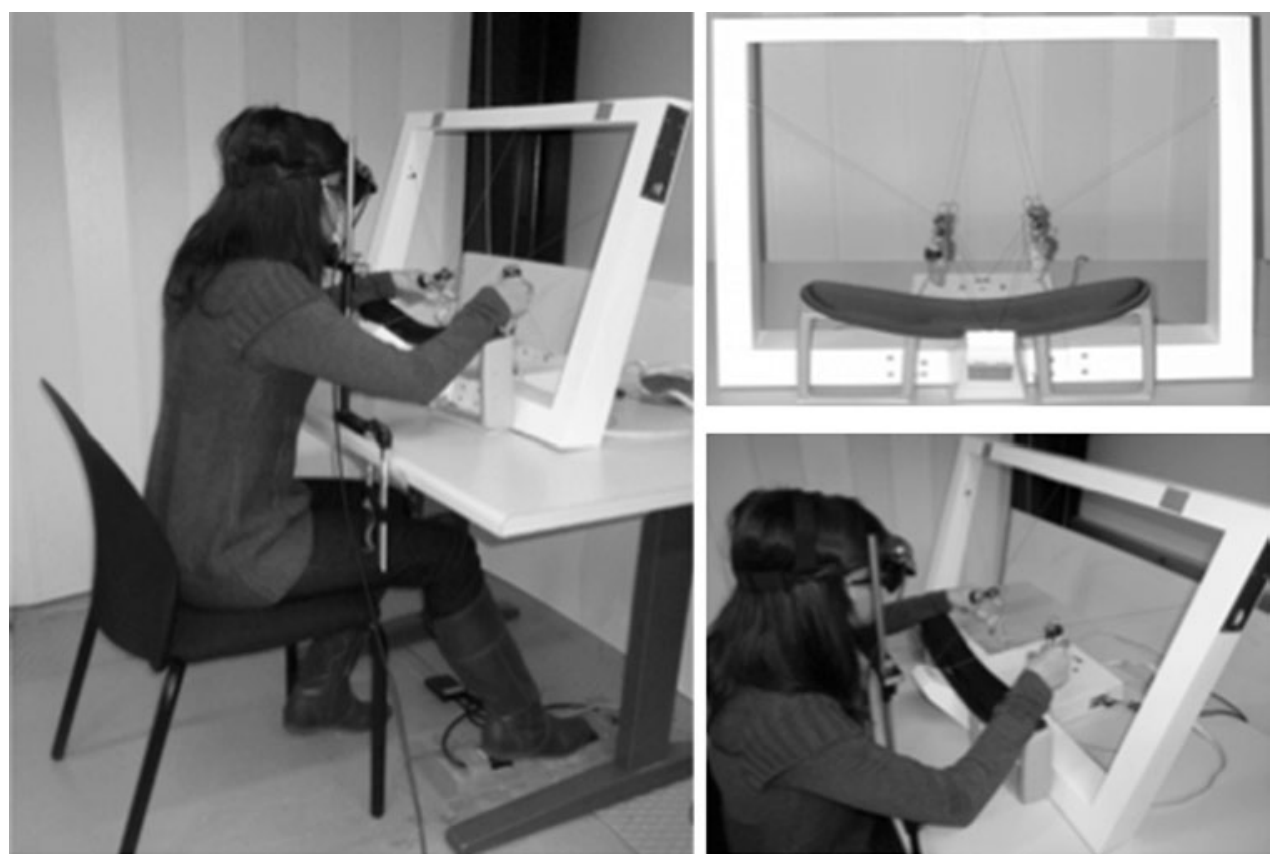
flexion and torsion. The spheres were attached to the nearest vertex of the mesh, and as the skeleton deforms, the shape of the mesh updates at every simulation cycle. Some of the fundamental classes of the CHAI3D platform were used to communicate between the haptic device and the computer. The universal device handler was used to automatically detect the available haptic devices. A haptic thread was used, which runs in higher priority to handle the high-speed servo loop between the simulation and the device. FFB was modeled in the haptic thread by using a spring force model. As the deformable object was grasped and pulled in any direction, a FFB modeled as a spring force field was provided in the opposite direction of pull. In this way as the proprioception cues of the subject were updated with pulling, a spring force field was given as feedback. The dynamic behavior of the deformable object was updated using the GEL dynamic engine in the haptic thread (Parikh et al. 2009).

Visual distractor stimuli were 5-mm-diameter virtual LEDs, positioned at the upper and lower claw of the robotic grippers. A visual fixation cross was presented between the vertical and horizontal midpoint of the corresponding four LEDs. Participants' responses were collected by using the same apparatus and procedures as described in Sengül et al. (2012).

In study 2, the same materials and apparatus were used as in study 1, except that a new virtual reality scene with a new task was used. A new soft deformable object was modeled to simulate a soft tissue test pad similar to the ones used in surgical training. The same open-source platform (CHAI 3D) and dynamic engine were used to model the deformable object and interactions. To manipulate the realism of the FFB, a delay in the FFB was implemented. Two semicircular surgical needles were modeled in the CAD program and imported into the virtual scene (see Fig. 5). Two big circular points were placed in the left and right upper part of the test pad to indicate the release point. Having a release point enabled a better control of the movement performed by the participants.

\section{Procedure}

\section{Study 1}

In the first experiment participants were seated in front of a table. The table height was adjusted for each subject. Participants held the two haptic interfaces in their left and right hands. In this experiment there were two main tasks: the tool-use task and the crossmodal congruency task. Instructions relating to these two tasks were given prior to testing.

In the tool-use task, participants used the robotic tools to interact with the deformable object for a duration of $40 \mathrm{~s}$. There were three conditions: "static", "without FFB" and

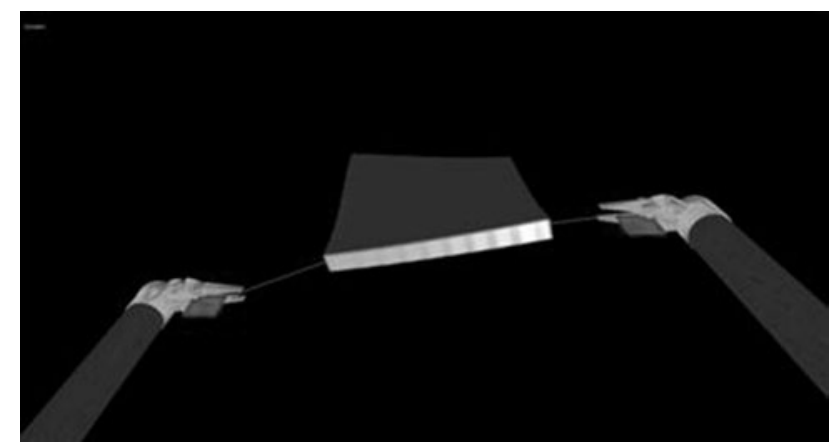

Fig. 2 Virtual reality view used in the tool-use task in experiment 1. Robotic tools were modeled based on the dimensions of the Da Vinci grasping tools. A deformable object was simulated, and red cubes indicated the grasping point. When the red cubes were pulled, a thread was shown to simulate the pulling of a thread in surgical procedures

"with FFB". In the "static" case, which was used as a baseline condition, participants did not move the robotic tool; they just held the tools for $40 \mathrm{~s}$. The static condition was included as a baseline condition, to compare the effects of merely holding the tools with active tool use with and without FFB. During the "without-FFB" condition, participants were required to use the tools to virtually grasp the boxes and pull them. While they were interacting with the boxes, the pulling of an elastic rope was visualized, but no FFB was provided during that interaction (see Fig. 2). In the "with-FFB" condition, while participants pulled the red boxes, in addition to the visualization of the pulling of an elastic rope as in the "without-FFB" condition, FFB was also provided. These three tool-use conditions were conducted in a random order. After participants performed one of the three tool-use tasks, they were instructed to perform the second task, the crossmodal congruency task.

In the crossmodal congruency task, participants were instructed to make speeded elevation discriminations of the vibrotactile stimuli. They were told that visual distractors would be presented just prior to the vibrotactile stimuli, but they should ignore them as much as possible. They were instructed not to close their eyes and fixate on the central fixation cross. In each trial, a visual distractor stimulus was presented $100 \mathrm{~ms}$ before the vibrotactile stimulus (SOA $100 \mathrm{~ms}$ ) (Shore et al. 2006; Sengül et al. 2012). Visual distractors were presented on the tip of each gripper's arm, matching the positions of vibrotactile stimuli on the participant's hands. The posture of the real hands was matched with the posture of the virtual grippers in order to increase the realism during the tool-use task and the CCE task. Subjects were told explicitly to match the posture of hands with the virtual grippers and not to move their hands during testing. The virtual grippers and the real hands matched not only in terms of posture but also in terms of their apparent 


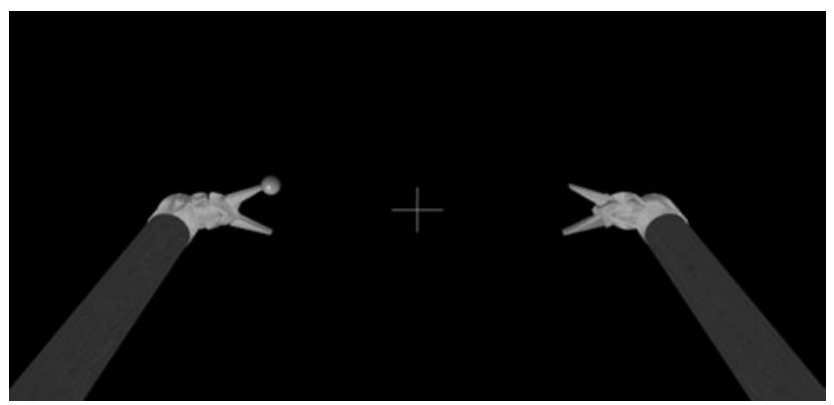

Fig. 3 Virtual reality view used in the CCE task in experiment 1. Following each tool-use task, a crossmodal congruency task was performed. A fixation cross was shown in the middle of the screen during the CCE task. Blue balls were presented as visual distractors at the tip of each gripper

spatial location. Apparent spatial location of the virtual tools was matched with the participants' hands by matching the distance between two virtual grippers with the distance between the participant's hands. This was monitored by the experimenter. Visual inspection during the CCE test confirmed that participants did not actively move their hands and they matched the posture of the hands and spatial location. The position of the grippers in the crossmodal congruency task is shown in Fig. 3.

The participant's right foot rested on the two pedals. Participants were instructed to press both of the pedals for the default condition, to lift the toes when they perceived vibrotactile stimuli at the index finger or lift the heel when they perceived it at the thumb. This toe/heel response was mapped to the index/thumb to make it compatible with the upper/lower elevation of the vibrotactile and visual stimuli. After every 16 CCE stimuli, they were informed that the task was finished and that they could prepare for the next tool-use task. Each block consisted thus of 16 CCE stimuli and was presented 8 times for each condition (static, without FFB and with FFB).

\section{Study 2}

The procedure of the experiment was similar to study 1 . There were again two main tasks: a tool-use task and the crossmodal congruency task. In the tool-use task, participants used the robotic surgical tools for $40 \mathrm{~s}$ to interact with the deformable object. There were two conditions: "synchronous" and "asynchronous," and they were presented in a random order determined by the computer. In the "synchronous" condition realistic feedback was given, and in the "asynchronous" condition unrealistic feedback was given. In the "synchronous" case, participants were required to grasp the needles and bring them to the release points, which were big balls on the upper part of the test pad. As they were pulling the needles, the pulling of an elastic rope was visually displayed with synchronous FFB. In the asynchronous case, participants performed the same task but with different FFB. As they pulled the needles, the pulling of an elastic rope was visualized synchronously, but the FFB was presented asynchronously, with a $600-\mathrm{ms}$ delay. After participants performed one of the two tool-use tasks for around $40 \mathrm{~s}$, they were instructed to perform the crossmodal congruency task, which was exactly the same as in study 1 .

Data analysis

Trials with incorrect responses were discarded from the reaction time analysis (but they were analyzed in the analysis of the percentage of errors). Trials with reaction times larger than 1,500 $\mathrm{ms}$ and above the 3-sigma boundaries were removed. Statistical analysis was based on the mean reaction times and error rates per condition for each participant. When vibrotactile stimuli and visual stimuli are presented at the same elevation, independent of relative side, it is referred to as "congruent". When they occur at different elevations, it is referred to as "incongruent". The CCE is defined as the difference in reaction times (or percentage error) between the incongruent and congruent conditions. Data from all trials that resulted in correct responses were analyzed by means of a repeated measures ANOVA on the mean values of reaction times. Paired $t$ tests were used for the post hoc comparison of the CCEs. All numerical values represent the mean and between-subject standard error. The results focused primarily on the RTs rather than errors, since it has been shown that RTs are more sensitive for the CCE (Pavani et al. 2000; Aspell et al. 2009; Shore et al. 2006; Sengül et al. 2012).

In study 1 and study 2, there were two blocks of 16 practice trials each, which were not analyzed. Participants' accuracy was more than $85 \%$ following the practice blocks. In study 1 , practice blocks were followed by 24 experimental blocks of 16 trials each, resulting in a total of 384 trials. Each of the 16 possible combinations of vibrotactile and visual distractor stimuli (4 visual distractors $\times 4$ vibrotactile target locations) was presented 8 times for each condition (static, without FFB and with FFB) in a pseudorandomized order determined by the computer. In study 2 practice blocks were followed by 20 experimental blocks of 16 trials each, with 10 blocks for synchronous FFB and 10 blocks for asynchronous FFB, resulting in a total of 320 trials. Each block was presented in a pseudorandomized order determined by the computer.

In sum, the first experiment was designed in a $2 \times 2 \times 3$ factorial manner. The 3 within-subject factors were "congruency" of the elevation of the vibrotactile stimuli with respect to the visual distractors (congruent vs. incongruent), the vibrotactile target "side" with respect to the visual 
Table 1 Mean reaction times in milliseconds and percentage of errors and mean crossmodal congruency effect for experiment 1 with standard errors in parentheses

\begin{tabular}{lccc}
\hline Feedback type & Congruent & Incongruent & CCE \\
\hline Experiment 1 & & & \\
Same side & & & \\
Static & & & \\
RT & $606.3(30.1)$ & $678.4(28.5)$ & $72.0(13.5)$ \\
$\%$ & $1.7(1.0)$ & $12.2(3.4)$ & $10.4(3.1)$ \\
Without FFB & & & \\
RT & $577.2(27.3)$ & $655.4(30.3)$ & $78.2(15.1)$ \\
$\%$ & $4.1(1.2)$ & $10.2(2.9)$ & $6.1(2.8)$ \\
With FFB & & & $113.1(18.3)$ \\
RT & $575.8(29.6)$ & $688.9(35.5)$ & $8.2(2.5)$ \\
$\%$ & $2.5(0.9)$ & $10.7(2.9)$ & \\
Different side & & & $35.5(13.9)$ \\
Static & & & $0.7(1.7)$ \\
RT & $625.6(31.7)$ & $661.2(28.2)$ & \\
$\%$ & $4.8(1.5)$ & $5.5(1.3)$ & $1.2(1.8)$ \\
Without FFB & & & $30.6(13.4)$ \\
RT & $603.4(31.4)$ & $640.5(32.7)$ & $3.5(1.9)$ \\
$\%$ & $6.1(1.8)$ & $7.1(1.6)$ & \\
With FFB & & & \\
RT & $605.6(35.4)$ & $636.2(30.7)$ & $(1.7)$ \\
$\%$ & $3.6(1.0)$ & & \\
\hline
\end{tabular}

distractor side (same vs. different) and the type of "condition" (static, without FFB and with FFB). Study 2 was designed in a $2 \times 2 \times 2$ factorial manner. The factors were "congruency" of the elevation of the visual distractors with respect to the vibrotactile stimuli (congruent/incongruent) and visual distractors "side" with respect to the vibrotactile stimuli side (same/different) and "delay" in FFB (synchronous/asynchronous) (Table 1).

\section{Results}

\section{Study 1}

Reaction times ranged between 411 and 891 ms. Statistical analysis performed on RTs revealed a main effect of congruency $(F(1,11)=32.9, p<0.001, d=0.749)$ and a significant interaction between the factors side and congruency $(F(1,11)=27.5, p<0.001, d=0.714)$, confirming that CCEs (i.e., the difference between incongruent and congruent visuo-tactile stimulation) were significantly larger in the same side conditions compared to the different side conditions $(t(1,11)=5.24 ; p<0.001)$. Crucially, we also found a three-way interaction between the factors condition, side and congruency $(F(2,11)=4.9, p<0.05$, $d=0.308)$. Post hoc analysis revealed that the same side CCEs in the with-FFB condition was significantly larger than same side CCE for the static condition $(t(1,11)=2.17$; $p<0.05)$ and the without-FFB condition $(t(1,11)=3.03$; $p<0.05)$, whereas no such difference between the static condition and the without-FFB condition ( $p=0.59$, N.S) and conditions for different side CCEs $(p>0.61)$ was found (see Fig. 4; Table 2). Since each experimental condition was presented in a random order, it is possible that the $\mathrm{CCE}$ in the static conditions was stronger in the last blocks, compared to the first ones, because they were preceded by blocks in which the tool was actively used. In order to control for the possible confound of carry-over effects in the static condition, we split the data of the static condition into two blocks (first four and last four blocks). Comparing the CCE values in the first and last blocks did not reveal any interaction of block with any of the other variables. Also, there was no significant difference between the first and last blocks ( $p=0.51$ N.S.), indicating that the comparable CCE magnitude in static and movement condition is not due to carry-over effects.

Statistical analysis performed on errors revealed a main effect of congruency $(F(1,11)=10.2, p<0.01, d=0.479)$ and a significant interaction between side and congruency $(F(1,11)=8.12, p<0.05, d=0.429)$. No other effects were found to be significant (all $p>0.21$ ). Comparisons between the ANOVA on RTs and errors revealed a common significant effect of congruency and a common interaction between congruency and side, suggesting no speed-accuracy trade-offs.

\section{Study 2}

Reaction times across the eight experimental conditions ranged between 491 and 899 ms. Statistical analysis performed on RTs revealed a main effect of congruency $(F(1,7)=43.2, p<0.001, d=0.861)$ and a significant two-way interaction between the factors side and congruency $(F(1,7)=20.3, p<0.01, d=0.743)$ with same side CCEs being significantly larger than different side CCEs $(t(1,7)=4.8 ; p<0.01)$. Crucially, we found a significant two-way interaction between congruency and delay $(F(1,7)=7.8, p<0.05, d=0.529)$. Post hoc comparisons revealed that the $\mathrm{CCE}$ in the FFB synchronous condition was larger than the CCE in the FFB asynchronous condition $(t(1,7)=2.8 ; p<0.05$; see Fig. 6; Table 2). In contrast to the three-way interaction in study 1 , we did not observe a significant three-way interaction in study 2 $(p=0.13$ N.S $)$. The three-way interaction in experiment 1 reflects a side-specific interaction between visuo-tactile cues, whereas the two-way interaction in experiment 2 refers to a general effect of visual distractors on tactile stimuli irrespective of the spatial side at which they were 


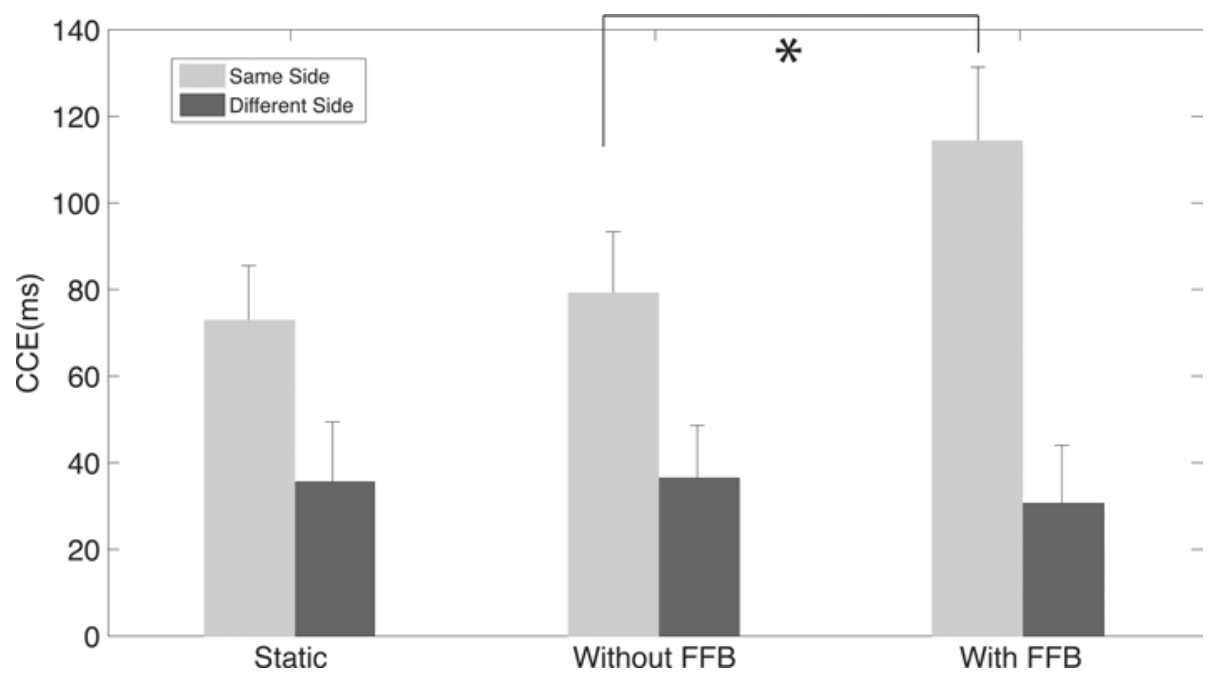

Fig. 4 Behavioral data from experiment 1 with the force feedback modulation. The graph shows the CCE (crossmodal congruency effect) in milliseconds in experiment 1 . The CCE was calculated by subtracting congruent from incongruent reaction times. Same side CCEs were significantly larger than the different side CCEs. More-

Table 2 Mean reaction times in milliseconds and percentage of errors and mean crossmodal congruency effect for experiment 2 with $\mathrm{SE}$ in parentheses

\begin{tabular}{lccc}
\hline Feedback type & Congruent & Incongruent & CCE \\
\hline $\begin{array}{l}\text { Experiment } 2 \\
\text { Same side }\end{array}$ & & & \\
Syn & & & \\
RT & $586.9(30.7)$ & $720.8(45.2)$ & $133.9(21.8)$ \\
$\%$ & $1.5(0.6)$ & $8.8(3.6)$ & $7.3(3.5)$ \\
Asyn & & & \\
RT & $625.2(37.0)$ & $721.6(49.4)$ & $96.4(17.4)$ \\
$\%$ & $1.4(0.6)$ & $11.4(4.7)$ & $10.0(4.6)$ \\
Different side & & & \\
Syn & & & \\
RT & $628.1(37.5)$ & $686.9(41.2)$ & $58.7(12.1)$ \\
$\%$ & $4.0(1.4)$ & $4.3(2.0)$ & $0.3(1.6)$ \\
Asyn & & & \\
RT & $630.9(38.2)$ & $673.9(40.0)$ & $43.0(7.1)$ \\
$\%$ & $1.3(0.6)$ & $5.4(1.8)$ & $4.1(1.3)$ \\
\hline
\end{tabular}

presented (no side/spatially specific effect). Additionally, the two-way interaction between the factors side and congruency indicates there is a CCE side effect (same side CCEs are larger than different side CCEs) irrespective of whether the FFB was delayed or not.

The ANOVA performed on errors revealed an interaction between side and congruency $(F(1,7)=5.85$, $p<0.05, d=0.454)$ and a tendency toward a main effect of congruency $(F(1,7)=4.77), p=0.065, d=0.402)$. over, the same side CCE for the FFB condition was significantly higher than the CCE for both the no-feedback and the static condition. Error bars represent standard errors

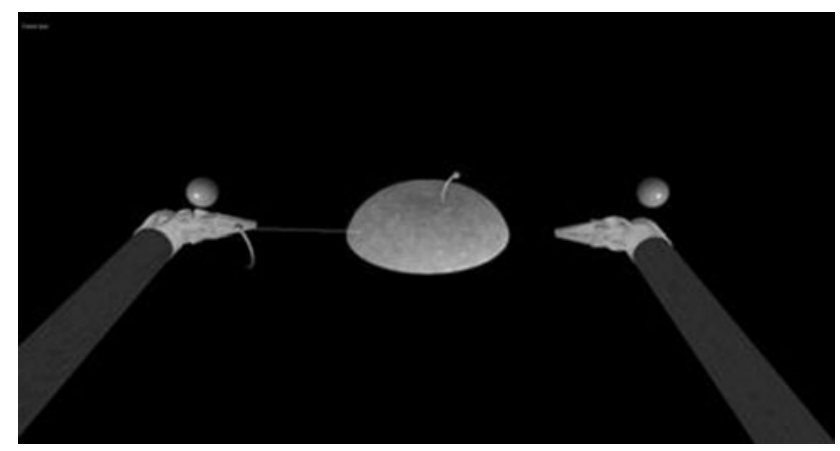

Fig. 5 Virtual reality view used in the tool-use task in experiment 2. A test pad was modeled to enable soft tissue deformation. Two surgical needles were shown, and participants were instructed to grasp and bring the needles to the release points that were shown with balls on the left and right side of the robotic tools

A comparison between the ANOVA on RTs and accuracy revealed the same tendency for a significant interaction between congruency and side, suggesting no speedaccuracy trade-offs. Task completion time, distance to the release point and position data were registered during the experiment and all conditions. For the synchronous FFB condition average distance to the release point was $1.9 \mathrm{~mm}$, average task completion time was $23.5 \mathrm{~s}$, and average deviation from the path was $18.3 \mathrm{~mm}$. The corresponding values for the asynchronous FFB condition were $1.9 \mathrm{~mm}$, $20.8 \mathrm{~s}$, and $19.2 \mathrm{~mm}$. There were no significant differences between the synchronous FFB and asynchronous FFB condition for the distance to the release point ( $p=0.22 \mathrm{~N} . \mathrm{S})$, task completion time ( $p=0.38$ N.S.) and deviation from 
Fig. 6 Behavioral data from experiment 2 with the delayed force feedback modulation. The graph shows the crossmodal congruency effect (CCE) in milliseconds in experiment 2 . The CCE was calculated by subtracting congruent from incongruent reaction times. Same side CCEs were significantly different than different side CCEs. Moreover, the same side CCE for the synchronous FFB condition was significantly higher than the CCE for the asynchronous FFB condition. Error bars represent standard errors

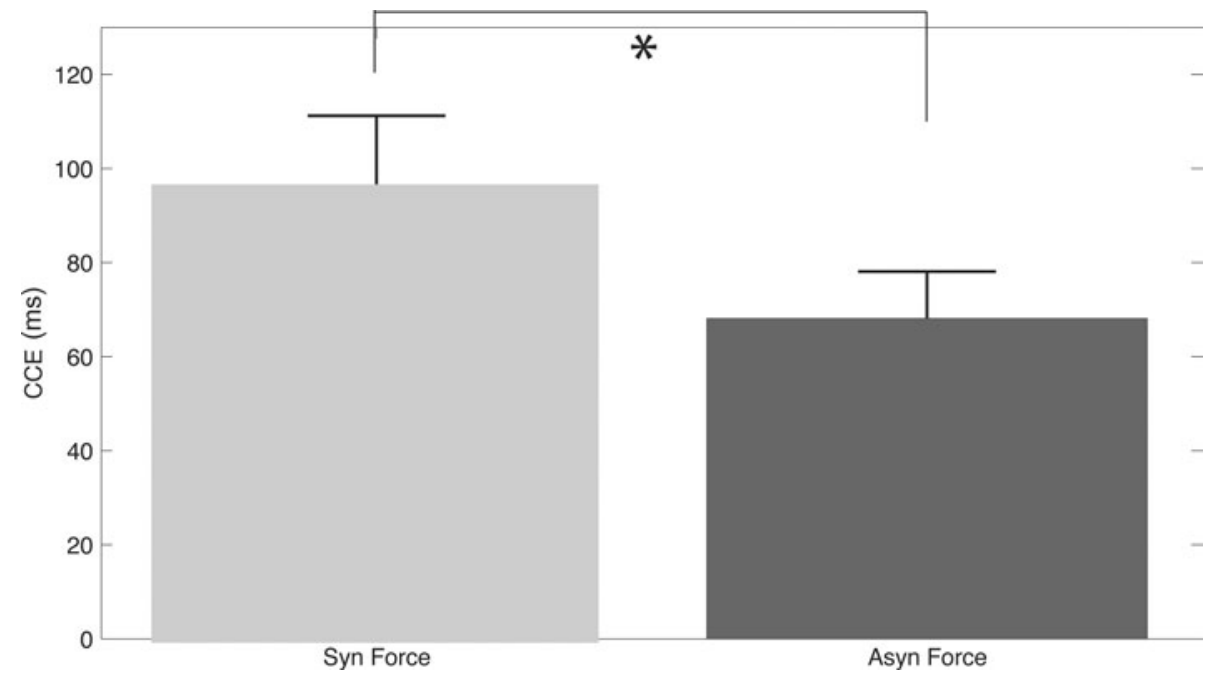

the path ( $p=0.56$ N.S.). Therefore, the FFB condition did not alter/increase task performance.

\section{Discussion}

In this study we examined the effect of FFB on visuotactile integration when using robotic surgical tools. First, we confirmed that there was an interaction between vision and touch, as reflected in the crossmodal congruency effect (CCE) for complex tools such as robotic manipulators. This finding extends studies on multisensory integration using a robotic setting with virtual hands (Rognini et al. 2012) and previous tool-use studies with physical tools (Maravita and Iriki 2004; Holmes 2012) and with virtual tools (Sengül et al. 2012). Second, we showed that the same side CCE was stronger after tool use with FFB as opposed to tool use without FFB. Hence, the spatial interaction between vision and touch is facilitated by realistic FFB related to the use of the tool. Third, CCEs were larger with synchronous FFB compared to asynchronous FFB, suggesting that realistic FFB enhances the incorporation of the robotic tool in the body representation only when the FFB is provided synchronously with the visual feedback.

The results of both experiments show that irrelevant visual distractors on the robotic tools interfered with tactile discrimination responses to stimuli applied to the participant's hand. The interfering effect of the visual stimuli on the robotic tools was reflected in slower RTs for incongruent compared to congruent visuo-tactile stimulation, which is known as the CCE. This finding of a CCE for a robotic tool is consistent with the previous studies on visuo-tactile integration in relation to the use of realworld tools (Holmes et al. 2004; Maravita et al. 2002). In addition, the results of both experiments also show larger CCEs when the visual distractors and vibrotactile stimuli were presented at the same azimuthal location (i.e., the same side CCE was larger than the different side CCE for all conditions in both experiments). As it has been suggested that the CCE side effect provides a direct measure of the strength of visuo-tactile interaction in peripersonal space (Holmes et al. 2004), the stronger same side CCE compared to a different side CCE suggests a possible modulation and extension of peripersonal space by robotic tool use. The results of experiment 1 showed that the spatial interaction between vision and touch depends on feedback type, which was reflected in a three-way interaction between congruency, side and feedback type. A post hoc analysis of this interaction revealed that there was no difference between the static condition and the without-FFB condition, but there was a difference between the withoutFFB and the with-FFB condition. This result shows that tool use without force feedback does not increase the difference between the same side and different side CCE, but that the CCE side effect is enhanced when FFB is present. Thus, virtual tool use without force feedback does not facilitate multisensory integration in peripersonal space. This could be due to the fact that in the present experiments with robotic tools participants were wearing HMDs and they did not view their real hands. This setup may have facilitated the automatic integration of visual information related to the robotic tool with proprioceptive signals related to the body-irrespective of whether the tool was actively used as in the without-FFB condition or not, as in the static condition (see also: Sengül et al. 2012). Additionally, during the CCE task, the posture of the real hands was matched with the posture of the virtual grippers in order to increase the correspondence between visual information related to the tool and proprioceptive information related to the position of the hands. Matching the position of the hands with the virtual grippers may have made the participants feel the visual distractor stimuli at 
the position of the hand, and this may have increased the interaction of the light stimuli with the vibrotactile stimuli.

The novelty of the present study lies in the systematic investigation of the effect of haptic FFB on multisensory integration. In the first experiment it was found that the spatial interaction between vision and touch depended on feedback type. More specifically, it was found that the same side CCE was largest for the FFB condition and it was significantly reduced for the other two conditions (static and without FFB). Moreover, there was also a greater difference between same side and different side CCEs in the FFB condition compared to the static and without-FFB conditions. The CCE side effect that was found in the FFB condition in experiment 1 and the general CCE effect that was found in both the synchronous and the asynchronous condition in experiment 2 likely reflect that visual stimuli were perceived in close proximity to the tactile stimuli applied to the hands. Thus, FFB enhances the remapping of visual information in peripersonal space. The enhanced CCE side effect likely reflects a stronger integration of the tool in the peripersonal space representation, as previous studies have shown that the size of the CCE is enhanced for stimuli that are easily integrated in the body representations (Rubber hands: Pavani et al. 2000; Tools: Maravita et al. 2002). Thus, the results of the first experiment show that visual stimuli at the end of the tools interfered more strongly with the vibrotactile stimuli on the handle when FFB (modeled as a spring force field which was provided in the opposite direction of pulling) was provided compared to when no FFB was available. This finding extends previous tool-use experiments, showing that multisensory visuo-tactile integration was facilitated by additional visuo-proprioceptive congruency (FFB) that might be considered as a case of more interactive or realistic tool use.

While the first experiment focused on how FFB changed visuo-tactile integration of a tool, the second experiment investigated whether the temporal congruency of FFB affected visuo-tactile integration. The second experiment served as a control study for the first experiment to study how FFB realism affects visuo-tactile integration. In experiment 2 it was found that the synchronous FFB resulted in a stronger overall CCE compared to the asynchronous FFB condition and that this was found irrespective of whether the visuo-tactile cues were presented on the same or different side. As it has been suggested that the magnitude of the CCE reflects the strength of an object's integration in the body representation (van Elk and Blanke 2011; Heed et al. 2012), this finding indicates that synchronous FFB enhances embodiment of the virtual tool. The delay in FFB resulted in an unrealistic FFB and as a consequence may have decreased the integration of the tool in the body representation, as reflected by a smaller CCE. This result is reminiscent of the findings by Pavani et al. (2000), who showed that the CCEs were smaller when rubber hands were placed in an anatomically impossible position, suggesting that the CCE provides a measure of hand ownership and body ownership (see also Aspell et al. 2009).

An important question is to what extent the data from the present study truly reflect an extension of peripersonal space, similar to the extension observed during real-world tool use. That is, in real-world tool use the graspable space of the hands is directly extended toward the area that would be out-of-reach without using a tool. In the present study participants viewed a virtual tool that was matched in its apparent spatial location to the position of the real hands. As such, the virtual tools may have been incorporated in the participant's body representation, without really extending peripersonal space (i.e., the virtual tools still operated in the same space as the participant's hands). However, we would like to point out that in surgical robotics it is precisely the process of tool incorporation that enables the extension of peripersonal space. For instance, in the Da Vinci system the surgeon views the tools at the felt location of his hands (resulting in the "incorporation" or "embodiment" of the tool; see Sengül et al. in prep.) while his peripersonal space is extended beyond his body to the location of the remote surgical robot. Similarly, in the present study, although the virtual tools were presented in the participant's peripersonal space, as virtual tools they can represent remote actions far beyond the space directly surrounding the subject's body. Thus, the present study provides direct insight into the different factors facilitating tool incorporation, which as such can facilitate the extension of peripersonal space.

In the present study we used a haptic device that has been described as an example of a "detached tool" according to the tool definitions of Holmes and Spence (2006). Detached tools, such as telesurgical tools, surgical simulators and haptic devices, enable neuroscientists to perform studies, which would not be possible with real tools, for instance as in the present experiments by introducing delays between performed and displayed movements and by modulating the FFB. Haptic perception combines both somatosensory and proprioception cues (Blanchard et al. 2011). Separating the contribution of tactile and proprioceptive cues is therefore experimentally difficult. Robotic systems such as haptic devices can be used to analyze the effect of these signals individually by introducing delays and manipulating FFB, thereby allowing more controlled experimental setups to analyze the effect of the different factors involved in multisensory integration in peripersonal space.

For the haptic community, learning how these tools are integrated in the brain's body representation may aid the design of ergonomic and usable interfaces and could enable a more objective assessment of their usability (Lee et al. 2005; Santos-Carreras et al. 2011). The results of the 
present studies show that methodologies from cognitive neuroscience (i.e., the crossmodal congruency task) can be used to investigate whether and how visual and tactile cues are integrated when using robotic technology. Up to now, human factors in robotics, especially in surgical robotics, have been quantified by means of questionnaires, surveys or performance-based studies (Prasad et al. 2002; Lee et al. 2005; Santos-Carreras et al. 2011). These studies focused on performance measures, but they neglected the basic and quantifiable change of multisensory integration due to tool use (Tan et al. 2010). Our methodology allowed us to study human factors in a current surgical interface with a more objective assessment technique by quantifying visuo-tactile integration based on reaction time and accuracy measures using the CCE.

\section{Conclusions}

In previous tool-use studies, researchers used physical tools to analyze the multisensory integration of vision and touch. These studies could not investigate the role of proprioceptive signals and their integration with visual and tactile cues because it is not possible to manipulate FFB systematically with physical tools. In the present study a haptic robot was used, thereby allowing us to manipulate FFB and to investigate the effect of visuo-proprioceptive signals on multisensory visuo-tactile integration in peripersonal space. We showed that online FFB facilitates the integration of vision and touch, as reflected in stronger CCEs. In addition, our results showed that the virtual tools might have been incorporated in the participant's body representation, without really extending peripersonal space. Similar mechanisms likely also apply to the use of robotic tools, such as those used in the field of surgical robotics, in which a surgeon may use bimanual haptic interfaces to control a surgery robot at a remote location (e.g., surgeon in New York controlling a robot in Paris, e.g., Sengül et al. in prep). Such robotically "extended" virtual scenarios provide novel research opportunities to understand whether such altered body representations reflect extensions or projections of peripersonal space (i.e., Holmes 2012; Maravita and Iriki 2004). Finally, such studies allow us to investigate whether and how the brain's representation of physical peripersonal space may differ from the representation of virtual-robotic peripersonal space.

\section{References}

Aspell JE, Lenggenhager B, Blanke O (2009) Keeping in touch with one's self: multisensory mechanisms of self-consciousness. PLoS One 4(8):e6488. doi:10.1371/journal.pone.0006488
Ayav A, Bresler L, Brunaud L, Boissel P (2004) Early results of one-year robotic surgery using the Da Vinci system to perform advanced laparoscopic procedures. J Gastrointest Surg Off J Soc Surg Aliment Tract 8(6):720-726. doi:10.1016/ j.gassur.2004.06.002

Bassolino M, Serino A, Ubaldi S, Ladavas E (2010) Everyday use of the computer mouse extends peripersonal space representation. Neuropsychologia 48(3):803-811. doi:10.1016/J.Neuropsycholo gia.2009.11.009

Blanchard C, Roll R, Roll JP, Kavounoudias A (2011) Combined contribution of tactile and proprioceptive feedback to hand movement perception. Brain Res 1382:219-229. doi:10.1016/ j.brainres.2011.01.066

Conti F, Barbagli F, Morris D, Sewell C (2005) CHAI 3D: an opensource library for the rapid development of haptic scenes. Paper presented at the IEEE world haptics, Pisa, Italy

Ehrsson H, Rosén B, Stockselius A, Ragnö C, Köhler P, Lundborg G (2008) Upper limb amputees can be induced to experience a rubber hand as their own. Brain 131(12):3443-3452

Farne A, Iriki A, Ladavas E (2005) Shaping multisensory action-space with tools: evidence from patients with cross-modal extinction. Neuropsychologia 43(2):238-248. doi:10.1016/j.neuropsycholo gia.2004.11.010

Graziano MS (1999) Where is my arm? The relative role of vision and proprioception in the neuronal representation of limb position. Proc Natl Acad Sci USA 96(18):10418-10421

Graziano MS, Gandhi S (2000) Location of the polysensory zone in the precentral gyrus of anesthetized monkeys. Exp Brain Res 135(2):259-266

Graziano MS, Reiss LA, Gross CG (1999) A neuronal representation of the location of nearby sounds. Nature 397(6718):428-430. doi:10.1038/17115

Graziano MS, Cooke DF, Taylor CS (2000) Coding the location of the arm by sight. Science 290(5497):1782-1786

Heed T, Backhaus J, Röder B (2012) Integration of hand and finger location in external spatial coordinates for tactile localization. J Exp Psychol Hum Percept Perform 38(2):386

Holmes NP (2012) Does tool use extend peripersonal space? A review and re-analysis. Exp Brain Res 218(2):273-282. doi:10.1007/ S00221-012-3042-7

Holmes NP, Calvert GA, Spence C (2004) Extending or projecting peripersonal space with tools? Multisensory interactions highlight only the distal and proximal ends of tools. Neurosci Lett 372(1-2):62-67. doi:10.1016/J.Neulet.2004.09.024

Holmes NP, Spence C (2006) Beyond the body schema. Human body perception from the inside out. Oxford University Press, Oxford, pp 15-64

Horgan S, Vanuno D (2001) Robots in laparoscopic surgery. J Laparoendosc Adv Surg Tech Part A 11(6):415-419. doi:10.1089/ 10926420152761950

Iriki A, Tanaka M, Iwamura Y (1996) Coding of modified body schema during tool use by macaque postcentral neurones. NeuroReport 7(14):2325-2330

Lee EC, Rafiq A, Merrell R, Ackerman R, Dennerlein JT (2005) Ergonomics and human factors in endoscopic surgery: a comparison of manual vs telerobotic simulation systems. Surg Endosc 19(8):1064-1070. doi:10.1007/S00464-004-8213-6

Maeso S, Reza M, Mayol JA, Blasco JA, Guerra M, Andradas E, Plana MN (2010) Efficacy of the Da Vinci surgical system in abdominal surgery compared with that of laparoscopy: a systematic review and meta-analysis. Ann Surg 252(2):254-262. doi:10. 1097/SLA.0b013e3181e6239e

Mahvash M, Hayward V (2004) High-fidelity haptic synthesis of contact with deformable bodies. IEEE Comput Graph Appl 24(2):48-55 
Marasco PD, Kim K, Colgate JE, Peshkin MA, Kuiken TA (2011) Robotic touch shifts perception of embodiment to a prosthesis in targeted reinnervation amputees. Brain 134(Pt 3):747-758. doi:10.1093/brain/awq361

Maravita A, Iriki A (2004) Tools for the body (schema). Trends Cognit Sci 8(2):79-86. doi:10.1016/J.Tics.2003.12.008

Maravita A, Spence C, Kennett S, Driver J (2002) Tool-use changes multimodal spatial interactions between vision and touch in normal humans. Cognition 83(2):B25-B34

Narazaki K, Oleynikov D, Stergiou N (2006) Robotic surgery training and performance: identifying objective variables for quantifying the extent of proficiency. Surg Endosc 20(1):96-103. doi:10.1007/s00464-005-3011-3

Okamura AM (2009) Haptic feedback in robot-assisted minimally invasive surgery. Curr Opin Urol 19(1):102-107. doi:10.1097/M OU.0b013e32831a478c

Parikh SS, Chan S, Agrawal SK, Hwang PH, Salisbury CM, Rafii BY, Varma G, Salisbury KJ, Blevins NH (2009) Integration of patientspecific paranasal sinus computed tomographic data into a virtual surgical environment. Am J Rhinol Allerg 23(4):442-447. doi:10. 2500/ajra.2009.23.3335

Pavani F, Spence C, Driver J (2000) Visual capture of touch: outof-the-body experiences with rubber gloves. Psychol Sci 11(5):353-359

Prasad SM, Maniar HS, Soper NJ, Damiano RJ, Klingensmith ME (2002) The effect of robotic assistance on learning curves for basic laparoscopic skills. Am J Surg 183(6):702-707

Rognini G, Sengül A, Aspell JE, Bleuler H, Blanke O (2012) Visuotactile integration and body ownership during self-generated action. Eur J Neurosci (in revision)

Rosen B, Ehrsson HH, Antfolk C, Cipriani C, Sebelius F, Lundborg G (2009) Referral of sensation to an advanced humanoid robotic hand prosthesis. Scand J Plastic Reconstr Surg Hand Surg /Nordisk plastikkirurgisk forening [and] Nordisk klubb for handkirurgi 43(5):260-266. doi:10.3109/02844310903113107
Santos-Carreras L, Hagen M, Gassert R, Bleuler H (2011) Survey on surgical instrument handle design: ergonomics and acceptance. Surg Innov. doi:10.1177/1553350611413611

Sengül A, van Elk M, Rognini G, Aspell JE, Bleuler H, Blanke O (2012) Extending the body to virtual tools using a robotic surgical interface: evidence from the crossmodal congruency task. PloS one

Serino A, Bassolino M, Farne A, Ladavas E (2007) Extended multisensory space in blind cane users. Psychol Sci 18(7):642-648. doi:10.1111/j.1467-9280.2007.01952.x

Shore DI, Barnes ME, Spence C (2006) Temporal aspects of the visuotactile congruency effect. Neurosci Lett 392(1-2):96-100. doi:10.1016/J.Neulet.2005.09.001

Spence C, Pavani F, Driver J (2004) Spatial constraints on visual-tactile cross-modal distractor congruency effects Cognitive. Affect Behav Neurosci 4(2):148-169

Stanney KM, Mourant RR, Kennedy RS (1998) Human factors issues in virtual environments: a review of the literature. Presence 7(4):327-351

Tan H, Reed C, Durlach N, Haptics K, Lafayette W (2010) Optimum information- transfer rates for communication through haptic and other sensory modalities. IEEE Trans Haptics 3(2):98-108

van Elk M, Blanke O (2011) The relation between body semantics and spatial body representations. Acta Psychol 138(3):347-358

Zimmerli L, Krewer C, Gassert R, Muller F, Riener R, Lunenburger L (2012) Validation of a mechanism to balance exercise difficulty in robot-assisted upper-extremity rehabilitation after stroke. J Neuroeng Rehabil 9:6. doi:10.1186/1743-0003-9-6

Zopf R, Savage G, Williams MA (2010) Crossmodal congruency measures of lateral distance effects on the rubber hand illusion. Neuropsychologia 48(3):713-725. doi:10.1016/j.neuropsycholo gia.2009.10.028 